\title{
AN INNOVATIVE APPLICATION ARCHITECTURE TO REDUCE CONTACT IN WORK ENVIRONMENTS
}

\author{
Aycan Vargün, Emin Tolgahan Polat, Özgür Macit and Uğur Serkan Taşkın \\ Research and Development Department \\ Koçtaş Construction Market Incorporated Company \\ Istanbul, Turkey
}

\begin{abstract}
COVID-19 has caused changes in working conditions and environments as well as in living conditions all over the world. The e-Health approach, on the other hand, can help employees to work in healthier environments with the appropriate use of information and communication technologies. In this paper, a solution architecture is presented to reduce the number of physical contacts that cause risk in work environments and to monitor the health status of employees. This architecture has been realized as a mobile application that employees can download on their phones. Usage results among employees have been included in the paper.
\end{abstract}

\section{KEYWORDS}

E-Health, COVID-19, Mobile Application, Java, Rest

\section{INTRODUCTION}

E-Health is a concept that aims to perform health transactions such as the realization of diagnosis and treatment procedures, clinical services, health data storage, patient registration and information tracking in electronic environment. It is getting more important day by day. Within the scope of E-Health, applications that provide decision support and reminders for users, electronic clinical management and communication tools, telemedicine applications for patient-doctor communication or communication between experts and applications that provide online training for healthcare personnel are acquiring significance every day (Toygar 2018).

There are many examples of e-health applications that touch on many different issues. The scope is expanding, from monitoring mother health by providing health questionnaires for postpartum depression (Jiménez-Serrano et al. 2015), to a variety of applications that offer blood glucose measurement and education for diabetic patients (Veazie et al. 2018). In addition, it is also possible to monitor health information with smart applications which use wearable sensor technology for various measurements such as heart rate, blood pressure, body temperature etc. It is also important that healthcare personnel and managers contribute with their time to design and develop new technologies (Royal Society 2006).

E-Health technologies can play an important role in COVID-19 test and indoor quarantine processes for COVID-19 positive patients with mild symptoms and can help for early intervention before these symptoms become severe (Adans-Dester et al. 2020).

Since COVID-19 affected working environments, monitoring the health of employees have become a very important issue to be addressed. For this purpose, this paper introduces a mobile application with its architecture in order to reduce contact caused by tools in common use. In addition, it controls the entries and exits of employees with the risk of COVID-19, by tracking contact records among employees and by monitoring their health status in the same environment.

The paper continues as follows: In section II, the problem definition is detailed, and a solution proposal is presented. In section III, the architectural infrastructure of the solution proposal is described with the necessary services and the technologies used. In section IV, the results of the application and future studies are presented. 


\section{PROBLEM DEFINITION AND SOLUTION PROPOSAL}

\subsection{Problem Description and Requirements}

The problem can be grouped under three headings. The first topic is that employees are using tools in common use at the starts and ends of their shifts in the working environment. A solution is required to eliminate these tools and to reduce contact. Secondly, monitoring health of employees is required. With the information obtained from this monitoring, it is necessary to prevent unhealthy employees from entering to the working area. Thirdly, all employees in contact with unhealthy employees should be determined. After the employees in health risk are identified, necessary steps should be taken by the responsible persons.

\subsection{Proposed Solution}

In Figure 1, A thorough application has been developed as a solution for the problem under three headings in Section 2.1. The key components of the application are four services at the backend, a mobile client and a web client using these services.

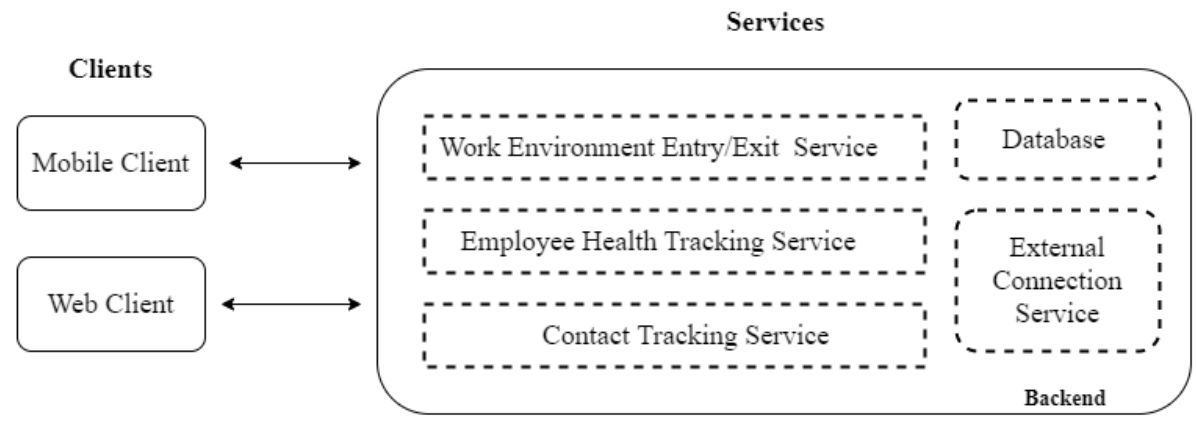

Figure 1. Key Architectural Components

\subsection{Solution Implementation and Results}

Java Spring Boot Framework has been used for the creation of Rest based APIs (Application Programming Interface) in the Backend. Spring Boot Framework is a stand-alone open-source framework produced by Pivotal Team, to develop microservices (Tutorialspoint 2021). An SQL server for database and the Hibernate/JPA (Java Persistence API) implementation for Java object mapping with tables in database are used. An SQL server is used since the data structure requires a relational database. A relational model is a model in which the data can be associated with each other (Codd 1970). JPA is a standard for associating Java objects with tables in a database. Hibernate is an implementation of this standard that performs object mapping (Javatpoint 2021).

Flutter SDK (Software Development Kit) has been used for the mobile client. Flutter SDK is a mobile software development environment developed by Google, which includes the Dart SDK that allows cross-platform application development and has a rich tool library (Flutter 2021). Html, CSS and JavaScript Fetch API for the web client is used to send requests to the backend services. Fetch API is a JavaScript interface that transmits Http/Https requests to the relevant server through the browser and transmits the returned responses to client (Mozilla 2021). The application backend is deployed on the Azure platform as an App Service and made available to employee users. Azure Web service is an HTTP-based Azure service that allows hosting web applications, REST APIs and mobile application backends by providing security, load balancing, and automatic scaling (Microsoft 2021). 


\subsubsection{Preparation of Backend Services}

- Employee Health Tracking Service: The purpose of this service is to obtain the daily health information of each employee and to convey this to other services. Employees are presented with a questionnaire containing pre-formed health questions by the institution doctor through the mobile interface. These survey questions can be delivered to the mobile client by creating and using the /getHealthQuestions API in Figure 2. The mobile client presents the questions to the users through the survey interface in its content. Employees' responses to the questionnaire are recorded daily in the database with the /postEmployeeAnswers API. The blocking information about the employees with health risk is added to the database by using BlockEmployeeIfUnhealty() function. This blocking information states that they cannot work that day.

The web client can receive blocking information on a list with /getBlockedEmployees or on an employee-based schema including health survey answers with /getBlockedEmployeeAnswers. The institution doctor can access the web client via browser and can obtain daily health information for the employees' health.

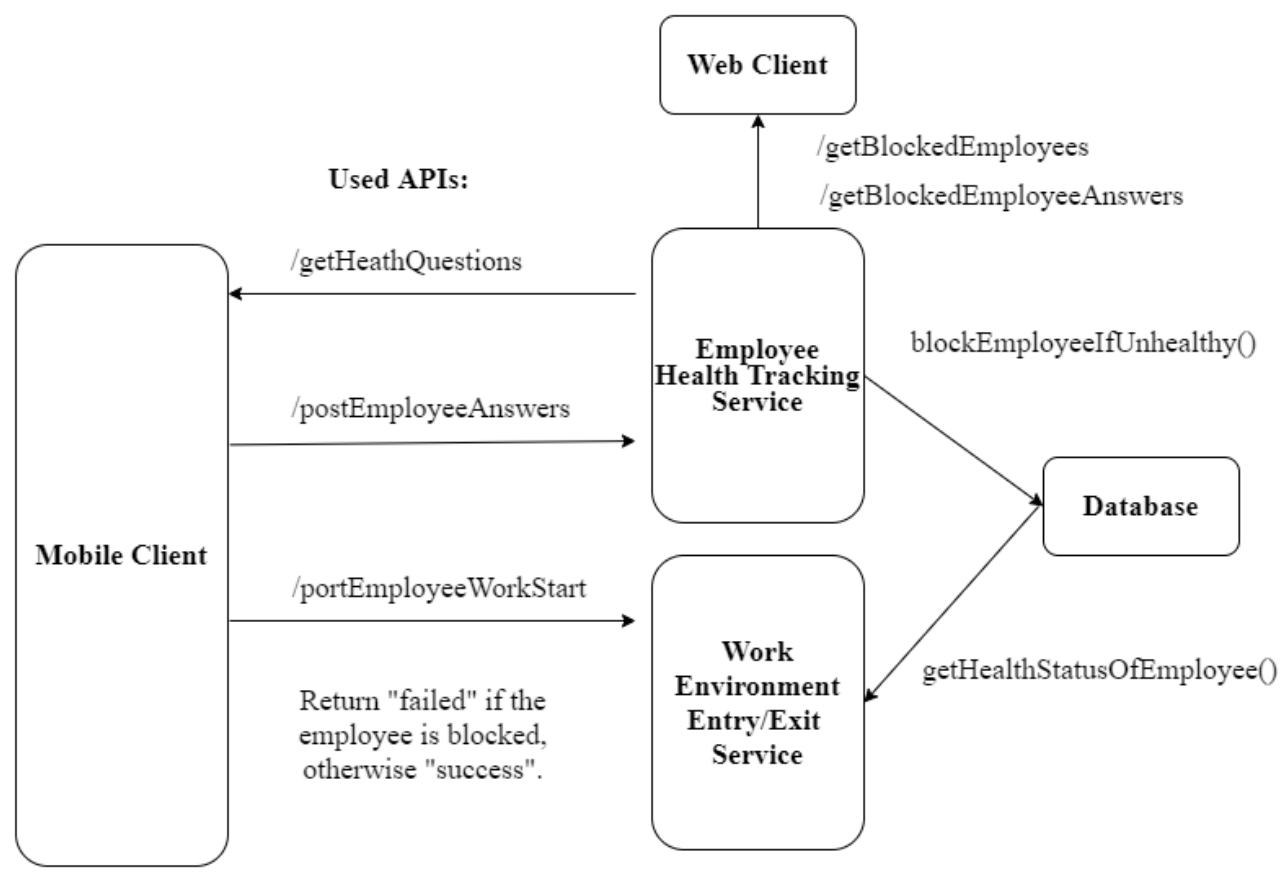

Figure 2. Working Environment Entry/Exit Service and Employee Health Monitoring Service

- $\quad$ Working Environment Entry/Exit Service: The main purpose of this service is to eliminate the contact by ensuring that the tools in common use while entering to the working environment are no longer needed. For this reason, special buttons are shown to the employees in the mobile interface so that they can report the start and the end of their shifts. There are two conditions that employees must meet to be able to make a report with these special buttons. The first is the GPS control that verifies that the employee is near the working environment. The second is whether he/she has a health risk. Mobile client receives GPS information on the phone and sends it to the backend via the /postEmployeeWorkStart API. This GPS information is compared with the actual GPS information of the working environment in the database. If the GPS information from the mobile client is closer than 100 meters to the actual GPS information of the working environment, the first verification has been achieved. Then, the database is checked with the getHealthStatusOfEmployee() function for employee health verification. If the employee has answered the health questionnaire of that day and no block has been added to the database for that employee in line with the answers, the second verification has also been achieved. The employee's shift report is logged, and "success" returns to the mobile client. If one of these verifications fails, "failed" is returned to the mobile client.

- Contact Tracking Service: The purpose of this service is to record the points where employees touch at the working area during the day, to identify all employees who touch the same point in case of a possible illness and to communicate with them with the approval of the institution doctor. 
A unique QR code was generated for each contact point and placed in the work area. Building, floor and room information are embedded to these $\mathrm{QR}$ codes. After that, employees who stand at a $\mathrm{QR}$ code point for more than a certain time must read this QR code from the mobile client and state that they are at that point. The mobile client sends the QR code to the backend with /postNewTouch API. This QR code is searched in the database and the point it belongs to is determined. Then, this record of reading QR code is saved to the database for that employee with the function addNewTouch(Employee, Checkpoint, TimeofContact).

The web client helps the institution doctor to identify the unhealthy employees and find the employees who have contacted the same points with these unhealthy employees. The filter on the web client interface requests employees at health risk using the /getEmployeesInRisk API. First, the employees who are blocked by giving risky answers to the health questionnaire are found by using the getUnhealtyEmployees() function. Second, QR code reading points that these employees come into contact with are obtained with the getRiskPoints(employees) function. These points are also used by the getEmployeesInRisk(riskPoints) function to find employees at risk who have touched the same points. The result is returned to the web client. In this way, the institution doctor obtains the list of employees at risk in Figure 3.

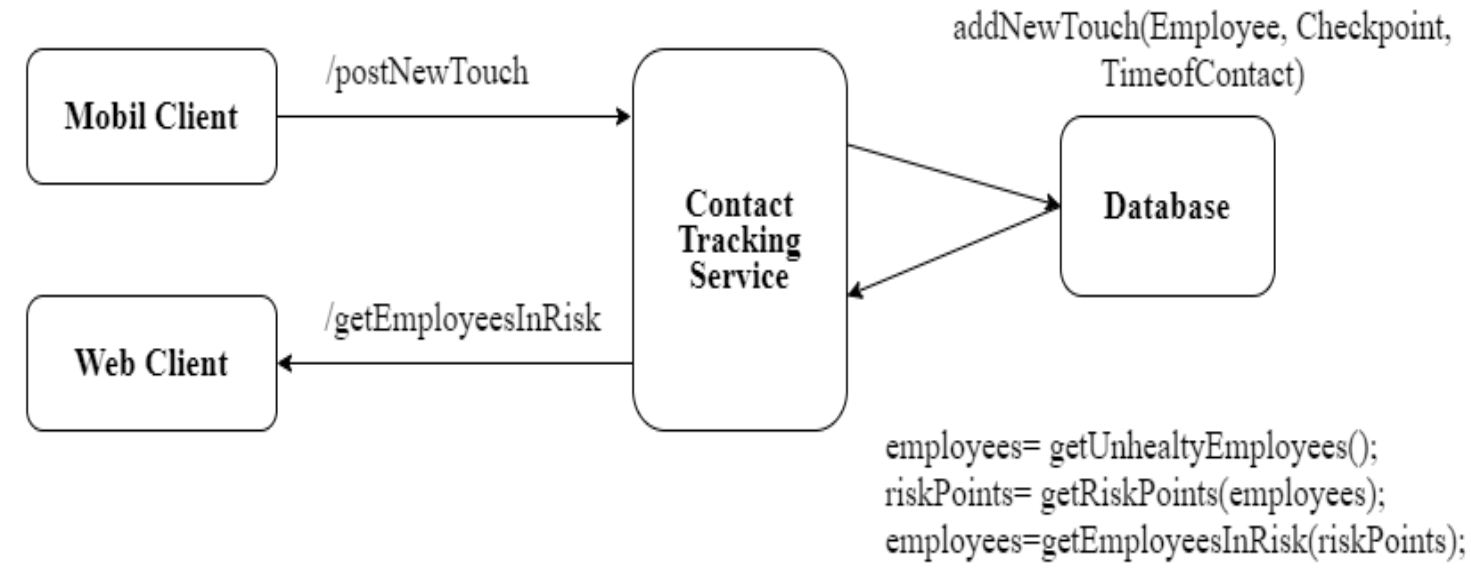

Figure 3. Contact Tracking Service

- Database and External Connection Services: This service is created to establish connection between the backend services and the external identity verification service for employees. This service speaks to the external authentication server for login.

\subsubsection{Preparing Clients}

- Mobile Client: First of all, screens are designed for each scenario. Second, a Flutter project is created by using the Flutter SDK. Next, models in line with APIs are created for requests of the Backend services. The functions to send requests and receive their responses have been added to Flutter project. Then the mobile application is uploaded to the Google Play Store and the IOS APP Store.

- Web Client: Web client is created by using Html, CSS and JavaScript. Through web client, the institution doctor can filter the employees according to their health status and can view the employees' responses to the health questionnaire with a time-based filtering. Web client requests are sent to the APIs in the backend by using the JavaScript Fetch API.

\subsubsection{Deployment Process}

A Resource Group has been created to put all application-related objects before the application backend is uploaded to Azure as an App service. An SQL database and SQL server have been created in this Resource Group. An App Insight has been created for logging and monitoring the application. Then, the backend is uploaded to the Resource Group as an App Service. After the deployment process has been completed successfully, the application backend has been serving the mobile and the web clients. 


\section{CONCLUSION}

\subsection{Usage Results}

The application has been used among employees since July 2020. The total number of users has reached to 1000 after January 2021. After the users login the application with their e-mail addresses, they answer the health questionnaire ever day to report their shifts. Employees who respond to the questions by stating that they don't pose any health risks can enter to the working environment. Those whose health status is at risk are blocked and cannot enter to the area.

On the left of the Figure 4, the health survey is shown on the mobile interface screen. The questions are health related like "Do you cough?", "Do you lose smell?" etc. When employees give a risky answer to the questionnaire, they are informed on a red screen (in the middle of the Figure 4) that they cannot make a report of shift. In addition, when they try to make a report of shift on the work reporting page (on the right of the Figure 4.), a warning is issued that they will not be able to report because their health might not be good.

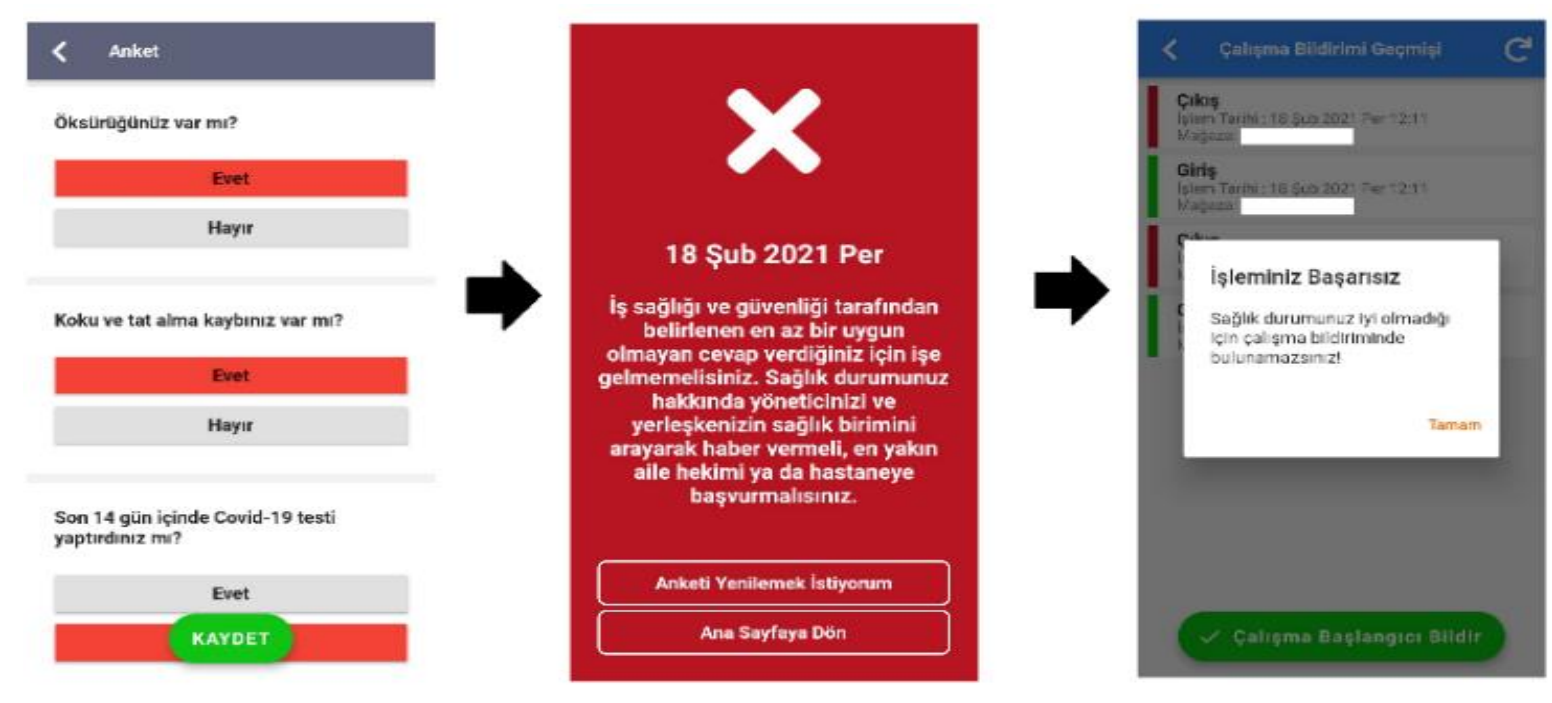

Figure 4. Working Environment Enter/Exit Report Scenario for Employees with Health Risks

Each QR reading transaction is recorded in the database. Employees can view the contact points they have read from the application. Since the contact points of the risky employees are recorded in the database, employees who touch the same points on the same day can also be determined. In Figure 5, there are two mobile client pages which belong to the Contact Tracking Service. On the left, the popup message which says "The reading operation is successful" is shown after a QR Code has been read by an employee. On the right, the employee's QR code history is listed. Three reads are listed. 


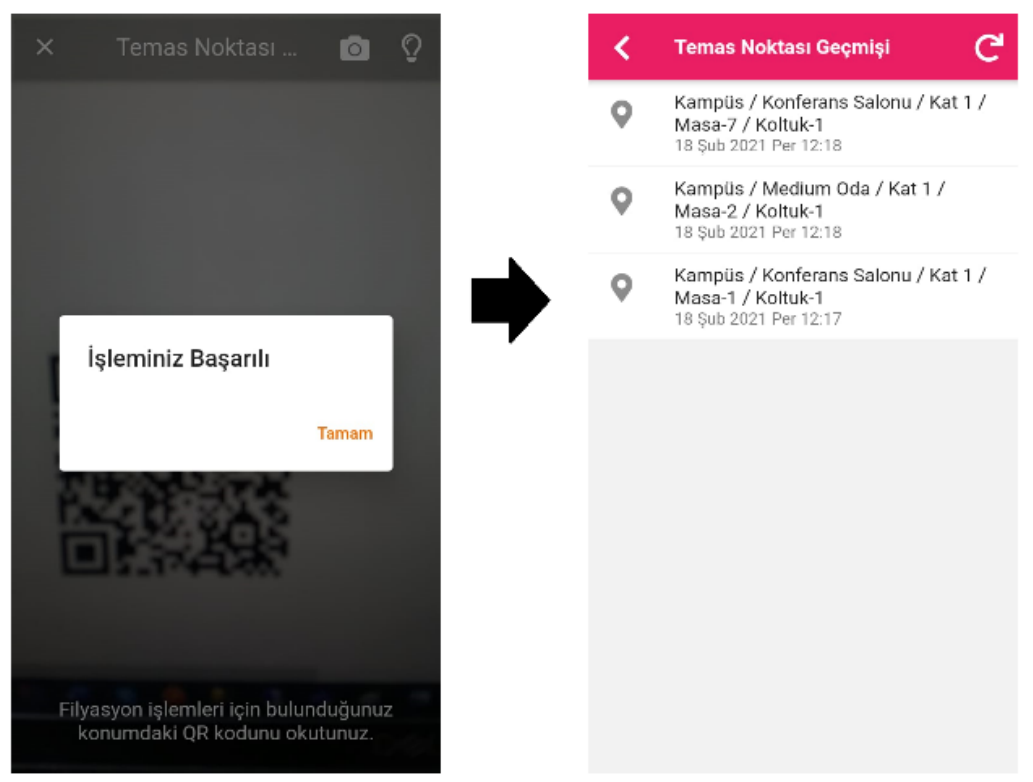

Figure 5. Touch Point QR Reading Process

The application has begun to run in July 2020. As seen in Table 1, the number of Entry/Exit reporting of employees by mobile application has increased in line with the increase in the number of employee users. The number of blocked people has reached its peak in October and November 2020. In the table, the row of "Blocked Employees Who Have Read Qrcodes" shows the number of blocked employees who have also read qrcodes within the same month in the working environment. As an example, if an employee is blocked in September 2020, and has read Qrcode in the same working environment in September 2020, he is added to the number in 9/20. The row "Number of People in Contact with The Blocked Persons" shows the number of the employees who have contacted within a month with the employees in the row "Blocked Employees Who Have Read Qrcodes". The number of blocked and contacted persons has reached its peak in November and December 2020. Thanks to the application, employees who have had risky contacts are identified and the opportunity to take action is provided.

Table 1. Working Environment Enter/Exit Report and Total UsageEnter/Exit Report and Total Usage

\begin{tabular}{l|l|l|l|l|l|l|l|l|l}
\hline Months: & $7 / 20$ & $8 / 20$ & $9 / 20$ & $10 / 20$ & $11 / 20$ & $12 / 20$ & $1 / 21$ & $2 / 21$ & $3 / 21$ \\
\hline Total Number of Users & 63 & 212 & 718 & 889 & 944 & 975 & 1144 & 1594 & 1967 \\
$\begin{array}{l}\text { Number of Entry/Exit Reporting of } \\
\text { Employees }\end{array}$ & 29 & 317 & 1303 & 7660 & 7304 & 5104 & 11564 & 13936 & 19406 \\
$\begin{array}{l}\text { Number of Blocked Employees } \\
\text { Blocked Employees Who Have Read } \\
\text { Qrcodes }\end{array}$ & 4 & 8 & 103 & 109 & 116 & 38 & 17 & 9 & 17 \\
$\begin{array}{l}\text { Number of People in Contact with } \\
\text { The Blocked Persons }\end{array}$ & 22 & 19 & 31 & 55 & 28 & 9 & 16 & 16 & 26 \\
\hline
\end{tabular}

In Figure 6, the graph shows the daily positive and negative questionnaires. The negative questionaries means that the employee health is risky per their answers to the health questions while the positives means that they are healthy. By the end of 2020, there is an increase in the number of positive questionnaires since the total users of the mobile application has increased as seen in Table 1. The number of negative questionnaires changes 1-10 each day. 


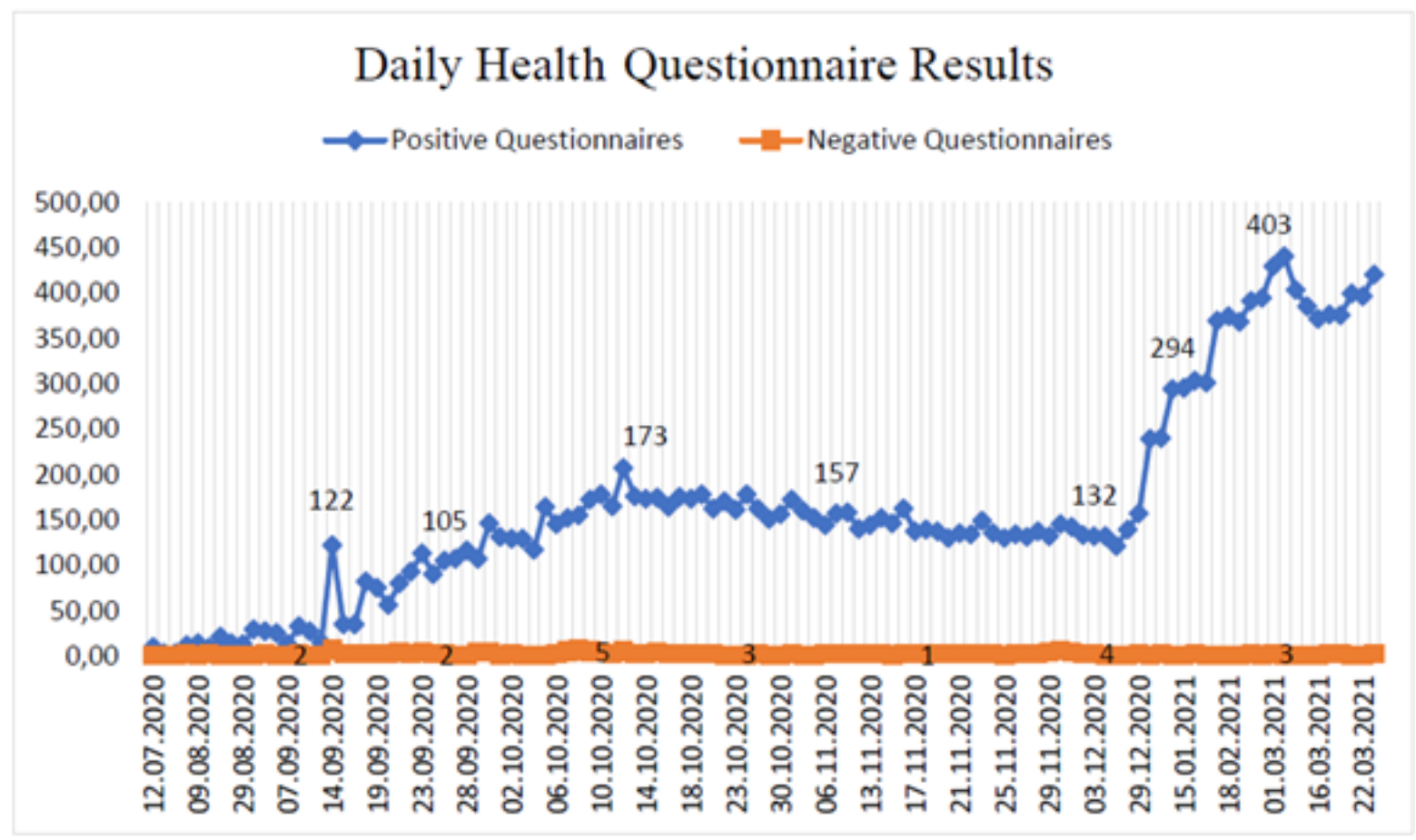

Figure 6. Daily Positive and Negative Questionnaires

\subsection{Future Developments}

Health monitoring of vaccinated employees, health training for employees and direct access to the institution doctor via the application as a telemedicine application are considered and planned for future developments.

\section{ACKNOWLEDGEMENT}

This work has been realized in the context of research and development works at Koçtaş Construction Market Incorporated Company.

\section{REFERENCES}

Adans-Dester, C.,P. et al., 2020. Can mHealth Technology Help Mitigate the Effects of the COVID-19 Pandemic?, In IEEE Open Journal of Engineering in Medicine and Biology, Vol. 1, pp. 243-248.

App service overview, Microsoft, viewed 27 March 2021, <https://docs.microsoft.com/en-us/azure/ app-service/overview/>.

Codd, E., F., 1970. A Relational Model of Data for Large Shared Data Banks, In Communications of the ACM, Vol. 13, No. 6, pp. 377-387.

Fetch API, Mozilla, viewed 27 March 2021, <https://developer.mozilla.org/en-US/docs/Web/API/Fetch_API>.

Jiménez-Serrano, S. et al, 2015. A Mobile Health Application to Predict Postpartum Depression Based on Machine Learning, In Telemedicine and e-Health, Vol. 21, No. 7, pp. 567-574.

JPA vs. Hibernate, Javatpoint, viewed 27 March 2021, <https://www.javatpoint.com/jpa-vs-hibernate>. 
Royal Society 2006. Digital Healthcare: The impact of information and communication technologies on health and healthcare, Royal Society, viewed 27 March 2021, <https://royalsociety.org//media/Royal_Society_Content/policy/publications/2006/8218.pdf >.

Spring Boot - $\quad$ Introduction, $\quad$ Tutorialspoint, $\quad$ viewed $27 \quad$ March 2021, <https://www.tutorialspoint.com/spring_boot/spring_boot_introduction.htm>.

Toygar, Ş., A., 2018. E-Sağlık Uygulamaları, In Yasama Dergisi, Vol. 37, pp. 101-123.

Veazie, S. et al, 2018. Rapid Evidence Review of Mobile Applications for Self-management of Diabetes, In Journal of General Internal Medicine, Vol. 33, pp. 1167-1176.

What is Flutter?, Flutter, viewed 27 March 2021, 〈https://flutter.dev/docs/resources/faq\#what-is-flutter>. 